\title{
Cinética de secagem e propriedades termodinâmicas de fatias de banana maçã BRS Tropical
}

\section{Drying kinetics and thermodynamic properties of BRS Tropical apple banana slices}

\section{Samuel Gonçalves Ferreira dos SANTOS ${ }^{1}$; Hélio Soares MIRANDA NETTO²; Dennis Ricardo Cabral CRUZ $^{3}$; Jefferson Kran SARTI" ${ }^{4}$ Vinícius Gonçalves ALMEIDA ${ }^{5}$; Renato Souza RODOVALHO ${ }^{6}$}

\author{
${ }^{1}$ Autor para correspondência, Mestrando em Fitotecnia, Universidade Federal de Viçosa, samuel-2100@hotmail.com \\ 2 Engenheiro Agrônomo, Instituto Federal Goiano - Campus Ceres, helionetto1@hotmail.com \\ ${ }^{3}$ Mestrando em Produção Vegetal, Universidade Federal de Goiás, denisribral@gmail.com \\ ${ }^{4}$ Engenheiro Agrônomo, Instituto Federal Goiano - Campus Ceres, jeffersonkran@gmail.com \\ ${ }^{5}$ Mestrando em Fitotecnia, Universidade Federal de Viçosa, almeidaagropec@gmail.com \\ ${ }^{6}$ Doutor em Agronomia, IF Goiano - Campus Ceres, renato.rodovalho@ifgoiano.edu.br
}

Recebido em: 28-05-2020; Aceito em: 30-08-2021

\begin{abstract}
Resumo
A banana é um produto biológico altamente higroscópico. Os produtos higroscópicos possuem a propriedade de sofrerem mudanças em seus teores de água, sob a forma de vapor ou líquido com o ambiente, por meio do processo de absorção ou dessorção. A secagem consiste na remoção de grande parte de água inicialmente contida no produto logo após a maturidade fisiológica, com o objetivo de promover longos períodos de armazenamento. Objetivou-se, neste trabalho, estimar as curvas de secagem de fatias de banana maçã da cultivar BRS Tropical, bem como obter o coeficiente de difusão efetivo e as propriedades termodinâmicas, em condições de secagem em bancada, sombra e sol. Aos dados experimentais foram ajustados diversos modelos matemáticos utilizados para representação da secagem de produtos agrícolas. O modelo matemático Midilli Modificado é o que melhor representa a cinética de secagem para as fatias de banana maçã BRS Tropical. O aumento da temperatura promove: uma taxa maior de remoção de água nas fatias de banana maçã durante a secagem; aumento do coeficiente de difusão efetivo sendo que esta relação pode ser descrita pela equação de Arrhenius; aumento da energia livre de Gibbs, enquanto a entalpia e a entropia decrescem.
\end{abstract}

Palavras-chave adicionais: desidratação; frutíferas; modelagem matemática;.

\begin{abstract}
The banana is a highly hygroscopic biological product. Hygroscopic products have the property of undergoing changes in their water content, in the form of vapor or liquid with the environment, through the process of absorption or desorption. Drying consists of removing much of the water initially contained in the product soon after physiological maturity, with the aim of promoting long periods of storage. The objective of this work was to estimate the drying curves of "apple" banana slices of the cultivar BRS Tropical, as well as to obtain the effective diffusion coefficient and thermodynamic properties, under bench, shade and sun drying conditions. To the experimental data, several mathematical models were used to represent the drying of agricultural products. The modified Midilli mathematical model is the one that best represents the drying kinetics for slices of BRS Tropical apple banana. The increase in temperature promotes: a higher rate of water removal in banana slices during drying; increase in the effective diffusion coefficient, this relationship can be described by the Arrhenius equation; increase in Gibbs free energy, while enthalpy and entropy decrease.
\end{abstract}

Additional Keywords: dehydration; fruit; mathematical modeling.

\section{Introdução}

A área de banana (Musa spp.) colhida no Brasil em 2017 foi de 469.471 hectares com uma produtividade de 6.764.324 toneladas (EMBRAPA, 2017). A fruta é comestível, muito nutritiva, sendo fonte de minerais como potássio, cálcio, fósforo, ferro, magnésio, bem como vitaminas. A banana apresenta longevidade altamente dependente da disponibilidade de água em seu interior, sendo que o incremento ou redução do teor de água pode prejudicar o armazenamento devido ao aparecimento de microrganismos e insetos (Bakhtavar et al., 2019).

A secagem consiste na remoção de grande parte de água inicialmente contida no produto logo após a maturidade fisiológica, com o objetivo de promover períodos mais longos de armazenamento, sem que ocorra perdas significativas durante o processo (Sousa et al., 2015). De acordo com Radünz et al. (2011), é de inegável importância o ajuste de diferentes modelos matemáticos aos dados experimentais de secagem na tomada de decisão, pois estes contribuem na melhoria da eficiência do processo. Além disso, os modelos 
matemáticos são ferramentas de engenharia úteis que podem facilitar o projeto, a otimização e a simulação do processo de secagem, como também, determinar a energia e o tempo requerido no processo (Kim \& Kim, 2017).

A energia de ativação é definida como a menor quantidade de energia necessária para que as moléculas de água ultrapassem as barreiras de energia durante a migração dentro de um produto (Morais et al., 2013). Por meio da energia de ativação é possível determinar as propriedades termodinâmicas dos alimentos que fornecem informações para projeção de equipamentos de secagem; para cálculo da energia requerida neste processo e para estudo das propriedades da água adsorvida (Corrêa et al., 2010, Viganó et al., 2012).

Diante do exposto, objetivou-se, neste trabalho, estimar as curvas de secagem de fatias de banana maçã da cultivar BRS tropical, bem como obter o coeficiente de difusão efetivo e as propriedades termodinâmicas, em condições de secagem de bancada, sombra e sol.

\section{Material e métodos}

O presente trabalho foi desenvolvido no Laboratório de Química Instrumental do Instituto Federal Goiano-Campus Ceres, município de CeresGO. Foram utilizados frutos de banana maçã da cultivar BRS Tropical, produzidas no próprio Campus, cujas coordenadas são: latitude $15^{\circ} 16^{\prime} 30^{\prime \prime}$ S e longitude $49^{\circ}$ 35' 54 " W.

A coleta dos frutos foi realizada nas primeiras horas da manhã, em setembro de 2018, após ausência de orvalho sobre suas superfícies visando evitar variações no seu teor de água inicial. Em seguida, as bananas foram selecionadas e transportadas para 0 Laboratório de Química Instrumental do Instituto Federal Goiano Campus-Ceres.

A determinação do teor de água inicial e final dos frutos de banana maçã da cultivar BRS Tropical foi realizada pelo método gravimétrico em estufa de circulação forçada, a $103 \pm 1{ }^{\circ} \mathrm{C}$, por $24 \mathrm{~h}$, em três repetições (ASABE, 2010). Já o teor de água de equilíbrio foi determinado pelo modelo de $G A B$, conforme recomendou Gouveia et al., (2004) para polpa de banana variedade prata. As bananas da variedade maçã BRS Tropical foram colhidas com teor de água de $75,01 \%$.

No laboratório, os frutos de banana foram descascados e fatiados em formato de chips, sendo colocados em Placas de Petri e, em seguida, divididos em quatro repetições para cada condição de secagem (bancada, sombra e sol). Um termômetro foi utilizado na bancada do Laboratório de Química Instrumental para aferir as temperaturas mínimas e máximas. As amostras foram pesadas em balança analítica com precisão de quatro casas decimais. Cada placa de Petri foi identificada e recebeu, em média 20 gramas de polpa de banana em formato chips, em espessura de aproximadamente de $0,0052 \mathrm{~m}$, sendo pesadas e levadas para o local de secagem. Realizou-se ao todo 20 pesagens para cada condição de secagem em estudo, sendo o intervalo de cada pesagem de 20 minutos. Durante a realização de cada pesagem, aferiuse também, a temperatura e a umidade relativa do ambiente.

A primeira situação de secagem foi a exposição direta ao sol; a segunda foi a exposição à sombra, e pôr fim a terceira, em bancada de laboratório.

Ao final da secagem, foi determinado o teor de água em base seca, e posteriormente, realizado o cálculo da razão do teor de água $(\mathrm{RX})$ durante os processos de secagem por meio da equação 1.

$R X=\frac{\left(X-X_{e}\right)}{\left(X_{i}-X_{e}\right)}$

Em que: $X$ - teor de água do produto, decimal b.s.; $X_{i}-$ teor de água inicial do produto, decimal b.s.; $X_{e}$ - teor de água de equilíbrio do produto, decimal b.s.

A partir do $\mathrm{RX}$ de cada condição de secagem, realizou-se 0 ajuste dos modelos matemáticos de regressão não-linear (Tabela1) aos dados experimentais obtidos.

Tabela 1 - Modelos de regressão não-linear utilizados neste estudo. Nonlinear regression models used.

\begin{tabular}{|c|c|c|}
\hline Designação do modelo & Modelo & $\mathrm{N}^{\circ}$ \\
\hline Aproximação da Difusão & $R X=a \exp (-k t)+(1-a) \exp (-k b t)$ & (2) \\
\hline Dois Termos & $R X=a \exp (-k t)+b \exp (-k 1 t)$ & (3) \\
\hline Exponencial de Dois Termos & $R X=a \exp (-k t)+(1-a) \exp (-k$ a $t)$ & (4) \\
\hline Henderson e Pabis & $R X=a \exp (-k t)$ & (5) \\
\hline Logarítmico & $R X=a \exp (-k t)+b$ & (6) \\
\hline Midili & $R X=a \exp \left(-k t^{n}\right)+b t$ & (7) \\
\hline Midili Modificado & $R X=\exp \left(-k t^{n}\right)+a t$ & (8) \\
\hline Newton & $R X=\exp (-k t)$ & (9) \\
\hline Page & $R X=e x p\left(-k t^{n}\right)$ & (10) \\
\hline Page Modificado & $R X=\exp \left(-\left(k t^{n}\right)\right)$ & (11) \\
\hline
\end{tabular}

Em que: $\mathrm{RX}$ - razão do teor de água do produto (adimensional); $\mathrm{t}$ - período de secagem, horas; k, k1, k2coeficiente de secagem; a, b, c e n - constantes empíricas dos modelos. 
$\mathrm{Na}$ seleção dos melhores modelos para representar a cinética de secagem de fatias de banana maçã da cultivar BRS tropical, foram considerados a significância dos coeficientes de regressão pelo teste $t$ a $p \leq 0.05$, a magnitude do coeficiente de determinação (R2), o erro médio relativo $(P)$ e o erro médio estimado (SE). Para a recomendação do modelo matemático, foi verificado o $R^{2}$ mais próximo à magnitude, valores de $P$ abaixo de $10 \%$ e os valores de SE mais próximos de zero. Os valores do erro médio estimado (SE) e o erro médio relativo $(P)$ foram calculados pelas equações 12 e 13 , respectivamente.

$\mathrm{SE}=\sqrt{\frac{\sum_{\mathrm{i}=1}^{\mathrm{n}}(\mathrm{Y}-\mathrm{Y})^{2}}{\mathrm{GLR}}}$

$\mathrm{P}=\frac{100}{\mathrm{n}} \sum_{\mathrm{i}=1}^{\mathrm{n}}\left(\frac{|\mathrm{Y}-\hat{\mathrm{Y}}|}{\mathrm{Y}}\right)$

Em que: SE - erro médio estimado; $\mathrm{n}$ - número de casos; $Y$ - valor experimental; $\hat{Y}$ - valor estimado; GLR - grau de liberdade do modelo (número de observações experimentais menos o número de coeficientes do modelo).

O coeficiente de difusão efetivo $\left(D_{\text {ef }}\right)$ foi obtido pelo ajuste do modelo da difusão líquida para forma geométrica de placa plana aos dados experimentais da secagem, com a aproximação de 8 termos, de acordo com a equação 14 (Brooker et al., 1992). A espessura e o diâmetro dos chips de polpa de banana maçã foram mensurados utilizando-se um paquímetro digital para obtenção de uma média para uso no modelo.

$$
R X=\sum_{n=1}^{\infty} \frac{4}{\lambda n^{2}} \exp \left(-\frac{\lambda n^{2}}{L^{2}} \operatorname{Def} T\right)
$$

Em que: $\mathrm{RX}$ - razão de teor de água, adimensional; $D_{\text {ef }}$ - coeficiente de difusão efetiva, $\mathrm{m}^{2} \mathrm{~s}^{-1} ; \mathrm{T}$ - tempo, $\mathrm{s} ; n$ número de termos; $\mathrm{L}$ - espessura dos produtos, $\mathrm{m}$; $\mathrm{e}$, $\lambda n$ - raízes da equação de Bessel de primeiro tipo e com ordem 0 .

A relação entre o coeficiente de difusão efetivo $\left(D_{\text {ef }}\right)$ e a temperatura do ar de secagem foi determinada pelo modelo de Arrhenius (equação 15).

$$
D_{e f}=D_{0} \exp \left[-\frac{E_{a}}{R T_{a}}\right]
$$

Em que: $\mathrm{D}_{0}$ - fator pré-exponencial, $\mathrm{m} \mathrm{s}^{-1} ; \mathrm{E}_{\mathrm{a}}$ - energia de ativação, $\mathrm{J} \mathrm{mol}^{-1} ; \mathrm{R}$ - constante universal dos gases, $8,314 \mathrm{~J} \mathrm{~mol}^{-1} \mathrm{~K}^{-1} ; \mathrm{T}_{\mathrm{a}}$ - temperatura absoluta, $\mathrm{k}$.

As propriedades termodinâmicas: entalpia específica, entropia específica e energia livre de Gibbs, relacionadas ao processo de secagem das fatias de banana maçã foram determinadas através do método descrito por Jideani \& Mpotokwana (2009).

$$
\begin{aligned}
& \Delta H=E_{a}-R T_{a} \\
& \Delta S=R\left[\ln \left(D_{0}\right)-\ln \left(\frac{k_{B}}{h_{P}}\right)-\ln \left(T_{a}\right)\right] \\
& \Delta G=\Delta H-T_{a} \Delta S
\end{aligned}
$$

Em que: $\Delta \mathrm{H}$ - entalpia, $\mathrm{J} \mathrm{mol}^{-1} ; \Delta \mathrm{S}$ - entropia, $\mathrm{J} \mathrm{mol}^{-1}$; $\mathrm{k}_{\mathrm{B}}$ - constante de Boltzmann, $1,38 \times 10^{-23} \mathrm{~J} \mathrm{~K}^{-1}$; $\mathrm{h}_{\mathrm{P}}-$ constante de Planck, 6,626×10-34 $\mathrm{J} \mathrm{s}^{-1}$.

\section{Resultados e discussão}

O teor de água inicial das fatias de banana maçã BRS Tropical foi de 3,0 (b.s.), sendo reduzido para $2,19,1,40$ e 0,92 (b.s.) no final do processo de secagem, para as condições de secagem em bancada, sombra e sol, respectivamente. As temperaturas para as condições: bancada, sombra e sol, foram de 28,5 \pm $1,01^{\circ} \mathrm{C} ; \quad 32,3 \pm 0,387^{\circ} \mathrm{C} ; \quad 42,8 \pm 4,83^{\circ} \mathrm{C}$, respectivamente. $O$ tempo de secagem foi de nove horas para todas as condições de secagem. Já a umidade relativa média durante 0 processo de secagem foi de $79,3 \%$, para a condição de bancada e $88,5 \%$, para as condições de sombra e sol. Como esperado, o tempo de secagem diminuiu com o aumento da temperatura (Figura 1), situação também observada por Leite et al. (2015), que realizaram a cinética de secagem de fatias de banana variedade Terra, nas temperaturas do ar de secagem de 40, 50 e $60^{\circ} \mathrm{C}$. De acordo com Goneli et al. (2014), a redução do tempo de secagem com o aumento da temperatura pode ser explicada devido ao aumento do gradiente de pressão de vapor entre o ar de secagem e o ar no interior do fruto.

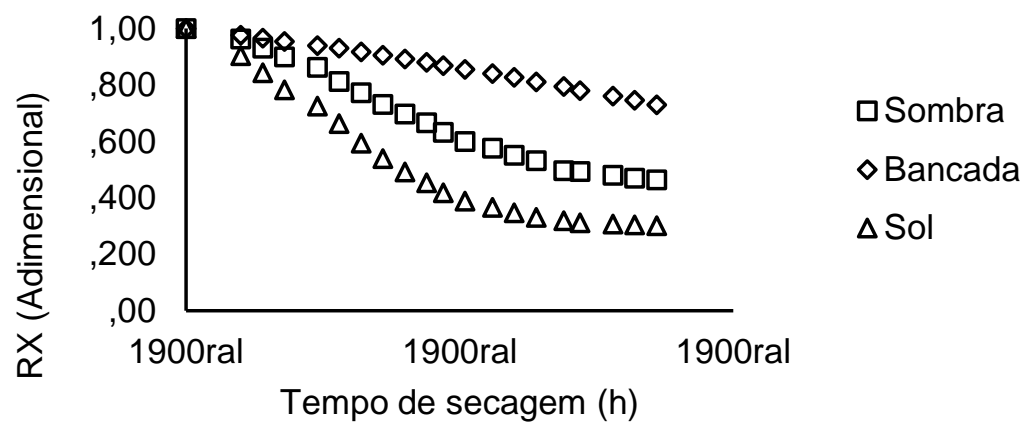

Figura 1 - Valores médios da razão do teor de água $(R X)$ de fatias de banana maçã tropical (decimal) em função do tempo de secagem (horas) em três condições de secagem (sombra, meia-sombra e sol). Average values of the moisture content ratio $(R X)$ of BRS Tropical "apple" banana slices (decimal) as a function of drying time (hours) in three drying conditions (bench, half shade and sun). 
Na Tabela 2 são apresentados os parâmetros estatísticos usados para comparar os 10 modelos testados para descrever a cinética de secagem dos chips de banana maçã BRS Tropical. Para as três condições, os modelos matemáticos ajustados aos dados experimentais apresentaram coeficiente de determinação $\left(R^{2}\right)$ superior a $95 \%$. Contudo, os modelos de Midilli e Midilli Modificado obtiveram os maiores valores de $\mathrm{R}^{2}$, com valores superiores a $98 \%$ para todas as condições. Segundo Karizaki (2016), valores mais altos de $\mathrm{R}^{2}$ indicam que o modelo teve bom ajuste aos dados experimentais. Todavia, o uso isolado deste parâmetro não é suficiente para caracterizar ajuste adequado de modelos não lineares (Corrêa et al., 2015), sendo necessário realizar uma análise aprimorada com outros parâmetros estatísticos (Zeymer et al., 2017). Logo, os valores obtidos do erro médio estimado $(\mathrm{SE})$ e erro médio relativo $(\mathrm{P})$ foram considerados.

Verifica-se, ainda na Tabela 2, que o modelo Newton foi o que apresentou maiores valores de P e SE e, consequentemente, não é recomendado. Já os modelos Midilli e Midilli Modificado foram os que mostraram para todas as condições de secagem, menores valores de SE e P. De acordo com Siqueira et al. (2012), quanto menores os valores de SE melhor o ajuste do modelo aos dados observados, enquanto Goneli et al., (2014) recomendam a análise conjunta de todas as temperaturas envolvidas no processo de secagem com erro médio relativo inferior a $10 \%$.

Em relação a distribuição de resíduos constatase que Midilli e Midilli Modificado apresentaram distribuição aleatória para as três condições de secagem, demostrando assim, serem adequados para descrever a secagem dos chips de banana maçã BRS Tropical (Tabela 2). Segundo Goneli et al. (2009), um modelo matemático deve apresentar distribuição aleatória para representar o processo de secagem de forma adequada, ou seja, os valores dos resíduos situam-se próximos à faixa horizontal em torno de zero.

Tabela 2 - Valores dos coeficientes de determinação ajustados $\left(R^{2}\right)$, dos erros médios estimados (SE), dos erros médios relativo $(\mathrm{P})$ e distribuição de resíduos (Dist) para os modelos matemáticos avaliados. Values of adjusted determination coefficients $\left(R^{2}\right)$, estimated mean errors $(S E)$, relative mean errors $(P)$ and residual distribution (Dist) for the mathematical models evaluated.

\begin{tabular}{|c|c|c|c|c|c|}
\hline Modelo matemático & $\begin{array}{c}\text { Condições } \\
\text { de secagem }\end{array}$ & $\left(\mathrm{R}^{2}\right)$ & SE (decimal) & $\mathrm{P}(\%)$ & Dist \\
\hline \multirow{3}{*}{ Aproximação por difusão } & Bancada & 99,60 & 0,034 & 0,457 & ${ }^{* *} \mathrm{~A}$ \\
\hline & Sombra & 96,23 & 0,262 & 4,619 & ${ }^{*} \mathrm{~T}$ \\
\hline & Sol & 97,46 & 0,254 & 5,686 & A \\
\hline \multirow{3}{*}{ Dois termos } & Bancada & 98,74 & 0,063 & 0,823 & $\mathrm{~T}$ \\
\hline & Sombra & 97,70 & 0,188 & 3,578 & $\mathrm{~T}$ \\
\hline & Sol & 97,32 & 0,254 & 7,176 & $\mathrm{~T}$ \\
\hline \multirow{3}{*}{ Exponencial de dois termos } & Bancada & 99,59 & 0,035 & 0,461 & $A$ \\
\hline & Sombra & 96,87 & 0,232 & 4,249 & $A$ \\
\hline & Sol & 96,79 & 0,298 & 7,636 & $\mathrm{~T}$ \\
\hline \multirow{3}{*}{ Henderson e Pabis } & Bancada & 98,24 & 0,076 & 0,994 & $\mathrm{~T}$ \\
\hline & Sombra & 96,97 & 0,234 & 4,108 & $\mathrm{~T}$ \\
\hline & Sol & 97,09 & 0,288 & 7,449 & $\mathrm{~T}$ \\
\hline \multirow{3}{*}{ Logarítmo } & Bancada & 99,07 & 0,052 & 0,671 & $\mathrm{~T}$ \\
\hline & Sombra & 96,99 & 0,234 & 4,129 & $\mathrm{~T}$ \\
\hline & Sol & 97,42 & 0,272 & 6,515 & $\mathrm{~T}$ \\
\hline \multirow{3}{*}{ Newton } & Bancada & 96,85 & 0,108 & 1,416 & $\mathrm{~T}$ \\
\hline & Sombra & 95,53 & 0,285 & 4,828 & $\mathrm{~T}$ \\
\hline & Sol & 96,78 & 0,300 & 7,507 & $\mathrm{~T}$ \\
\hline \multirow{3}{*}{ Midilli } & Bancada & 99,62 & 0,032 & 0,425 & $A$ \\
\hline & Sombra & 98,44 & 0,153 & 2,912 & $A$ \\
\hline & Sol & 99,18 & 0,145 & 3,663 & A \\
\hline \multirow{3}{*}{ Midilli Modificado } & Bancada & 99,62 & 0,032 & 0,424 & $A$ \\
\hline & Sombra & 98,43 & 0,153 & 2,907 & $A$ \\
\hline & Sol & 99,18 & 0,144 & 3,653 & A \\
\hline \multirow{3}{*}{ Page Modificado } & Bancada & 99,53 & 0,039 & 0,507 & $A$ \\
\hline & Sombra & 97,03 & 0,227 & 4,153 & $\mathrm{~T}$ \\
\hline & Sol & 96,91 & 0,289 & 7,605 & $\mathrm{~T}$ \\
\hline \multirow{3}{*}{ Page } & Bancada & 99,53 & 0,039 & 0,507 & $A$ \\
\hline & Sombra & 97,03 & 0,227 & 4,153 & $\mathrm{~T}$ \\
\hline & Sol & 96,91 & 0,289 & 7,605 & $\mathrm{~T}$ \\
\hline
\end{tabular}

${ }^{\star}$ T- Comportamento tendencioso da distribuição dos resíduos; ${ }^{* \star} \mathrm{A}$-Comportamento aleatório da distribuição dos resíduos.

Portanto, ambos os modelos, Midilli e Midilli Modificado são recomendados para representar a

cinética de secagem dos chips de banana maçã. No entanto, por apresentar menores valores de $\mathrm{P}$ em 
relação a Midilli, o modelo Midilli Modificado foi escolhido.

$\mathrm{Na}$ Tabela 3 encontram-se os valores dos coeficientes do modelo Midilli Modificado para as diferentes situações de secagem. Constata-se que o coeficiente "k" aumentou com o aumento da temperatura, enquanto o coeficiente " $n$ " reduziu. Já o coeficiente "a" não apresentou tendência de comportamento. Todos os coeficientes foram significativos a $5 \%$ de probabilidade pelo teste t. De acordo com Babalis \& Belessiotis (2004), o coeficiente (k), que representa as condições externas de secagem, pode ser utilizado como uma aproximação para caracterizar o efeito da temperatura e está relacionado com a difusividade efetiva no processo de secagem no período decrescente e à difusão líquida que controla o processo.

Tabela 3 - Coeficientes do modelo Midilli Modificado para as diferentes situações de secagem de fatias de banana maçã tropical. Coefficients of the Modified Midilli model for the different drying situations of BRS Tropical "apple" banana slices.

\begin{tabular}{lccc}
\hline Condições de Secagem & \multicolumn{3}{c}{ Coeficientes do modelo Midilli Modificado } \\
\hline Bancada $\left(28,5 \pm 1,01^{\circ} \mathrm{C}\right)$ & $\mathrm{k}$ & $\mathrm{n}$ & $\mathrm{a}$ \\
Sombra $\left(32,3 \pm 0,387^{\circ} \mathrm{C}\right)$ & $0,0016^{*}$ & $1,8503^{*}$ & $-0,0215^{*}$ \\
Sol $\left(42,8 \pm 4,83^{\circ} \mathrm{C}\right)$ & $0,0715^{*}$ & $1,5365^{*}$ & $0,0373^{*}$ \\
\hline
\end{tabular}

${ }^{*}$ Significativo a $5 \%$ de probabilidade pelo teste t.; a, n, e k - coeficientes de ajuste dos modelos de Midilli Modificado.

$\mathrm{Na}$ Figura 2 verificam-se as curvas de secagem obtidas a partir do modelo Midilli Modificado para as diferentes condições de secagem estudadas. Constata-se que o modelo Midilli Modificado foi capaz de simular as diferentes condições de secagem de forma satisfatória, adequando-se bem aos dados experimentais. De acordo com Goneli et al. (2014) o melhor ajuste do modelo de Midilli aos dados experimentais de secagem está ligado, provavelmente, à rápida perda de água nos estádios iniciais do processo gerando uma curva de secagem mais acintosa e mais bem caracterizada matematicamente por este modelo. Este fato pode ser atribuído a produtos com elevados teores de água como é o caso das frutíferas.

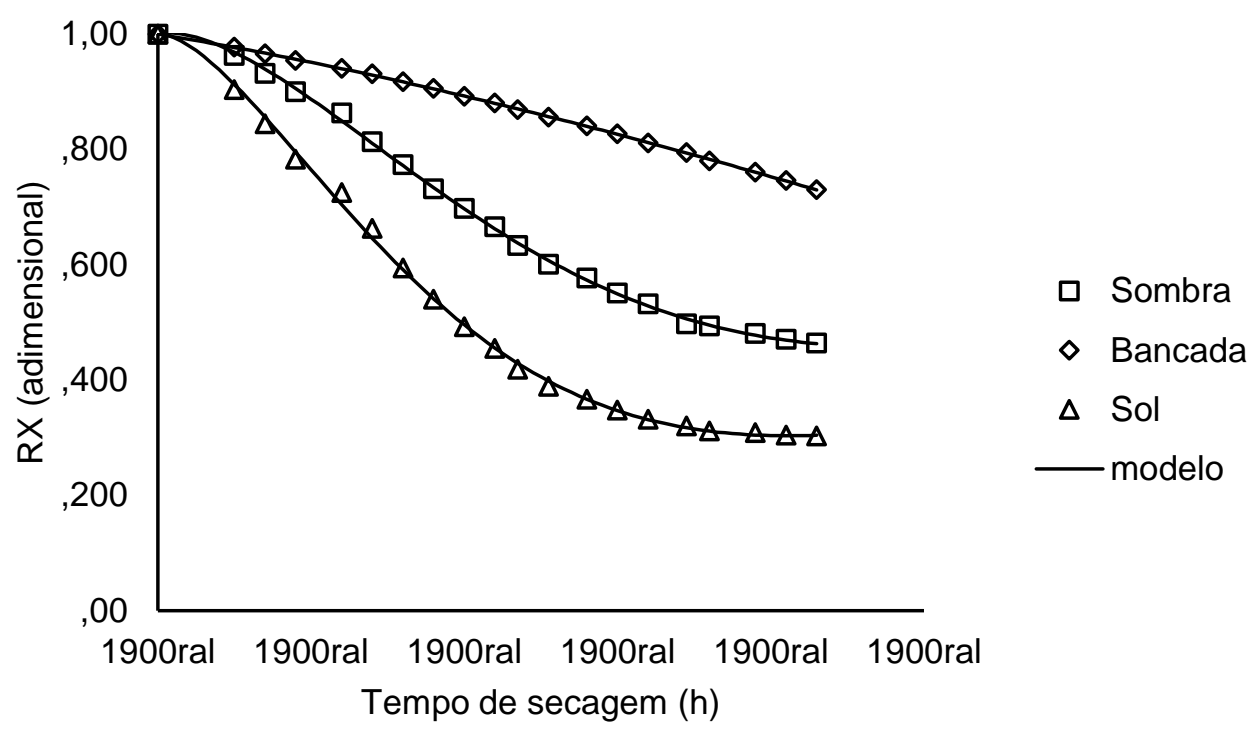

Figura 2 - Curva de secagem de fatias de banana maçã tropical ajustadas ao modelo de Midilli Modificado submetidas a diferentes situações de secagem. Drying curve of BRS Tropical "apple" banana slices adjusted to the Modified Midilli model subjected to different drying situations.

$\mathrm{Na}$ Figura 3 verificam-se os valores médios do coeficiente de difusão efetivo $\left(D_{\mathrm{ef}}\right)$ em $\mathrm{m}^{2} \mathrm{~s}^{-1}$ para as fatias de banana maçã tropical para as três condições de secagem estudadas. Observa-se que durante a secagem, 0 D ef aumentou com a elevação da temperatura apresentando valores de $2,80 \times 10^{-11}$; $1,61 \times 10^{-10} ; 3,53 \times 10^{-10} \mathrm{~m}^{2} \mathrm{~s}^{-1}$ para bancada, sombra e sol, respectivamente. Os resultados apresentados são 
coerentes com Jangam et al. (2010), na qual os produtos agrícolas geralmente apresentam valores de $D_{\text {ef }}$ entre $10^{-13}$ a $10^{-7}$. Ainda Jangam et al. (2010) citam que os valores da Difusividade para banana, nas temperaturas de 20 a $40^{\circ} \mathrm{C}$, variam de $3,0 \times 10^{-13}$ a
$2,1 \times 10^{-10} \mathrm{~m}^{2} \mathrm{~s}^{-1}$. Outros autores, como Leite et al. (2015), que trabalharam com fatias de banana variedade Terra, encontraram valores semelhantes com o presente trabalho, variando de $5,64 \times 10^{-10}$ a $9,86 \times 10^{-10}$, na faixa de temperatura de 40 a $60^{\circ} \mathrm{C}$.

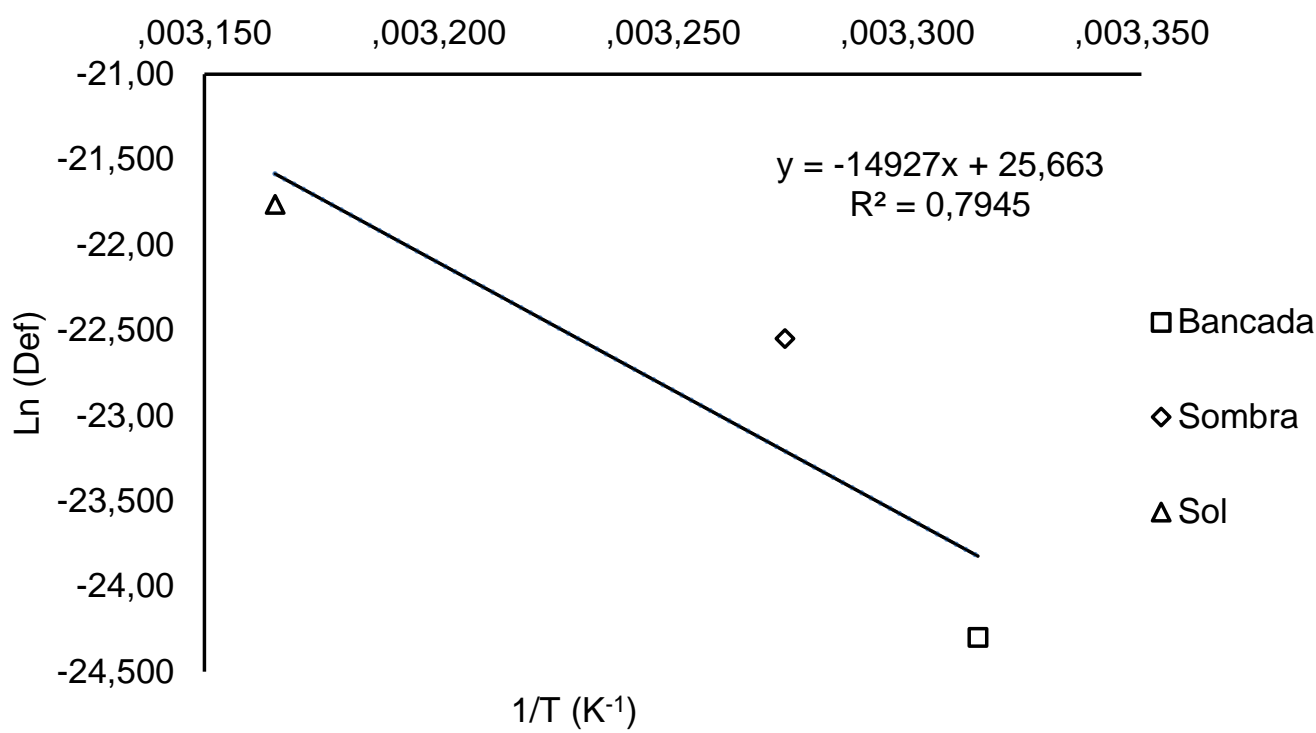

Figura 3 - Representação de Arrhenius para o coeficiente de difusão efetivo, em função da temperatura do ar, durante a secagem de fatias de banana maçã tropical. Representation of Arrhenius for the effective diffusion coefficient, as a function of air temperature, during the drying of BRS Tropical "apple" banana slices.

A energia de ativação (Ea) para o modelo da difusão líquida dos chips de banana maçã BRS Tropical foi de $85,266 \mathrm{~kJ} \mathrm{~mol}^{-1}$. Corrêa et al. (2007) afirmaram que a Ea consiste na facilidade com que as moléculas de água superam a barreira de energia durante a migração no interior do produto, sendo que: quanto menor a Ea, maior será a difusividade da água no produto durante 0 processo de secagem. Leite et al. (2015) encontraram valores de $24,07 \mathrm{~kJ}$ $\mathrm{mol}^{-1}$ para banana variedade terra nas temperaturas de 40 a $60^{\circ} \mathrm{C}$. Os valores encontrados no presente estudo diferem deste, possivelmente pelas diferentes condições de secagem. Leite et al. (2015) utilizaram secador descontínuo de bandejas com temperaturas do ar de secagem de $40^{\circ} \mathrm{C}, 50^{\circ} \mathrm{C}$ e $60^{\circ} \mathrm{C}$ e velocidade de $1 \mathrm{~m} \mathrm{~s}^{-1}$, diferenciando do presente trabalho na qual a secagem foi feita em ambiente natural (bancada, sombra e sol).
$\mathrm{Na}$ Tabela 4 verificam-se as propriedades termodinâmicas para os chips de banana maçã BRS Tropical nas situações de secagem estudadas. A entalpia $(\Delta \mathrm{H})$ reduziu com o aumento da temperatura e os menores valores de entalpia indicam menor energia necessária para remover a água livre do produto durante a secagem (Oliveira et al., 2010). O presente trabalho apresentou, como esperado, menor valor de entalpia para temperaturas mais elevadas de secagem, indicando que menor quantidade de energia é requerida para que a secagem ocorra em temperaturas mais altas. Os valores de entalpia para o processo de secagem foram de 82,7535; 82,7219; e 82,6346 $\mathrm{kJ} \mathrm{mol}^{-1}$ para as situações de secagem bancada, sombra e sol, respectivamente.

Tabela 4. Propriedades termodinâmicas do processo de secagem de fatias de banana maçã tropical: entalpia específica $(\Delta \mathrm{H})$, entropia específica $(\Delta \mathrm{S})$ e energia livre de Gibbs $(\Delta \mathrm{G})$. Thermodynamic properties of the drying process of BRS Tropical "apple" banana slices: specific enthalpy $(\Delta H)$, specific entropy $(\Delta S)$ and Gibbs free energy $(\Delta G)$.

\begin{tabular}{ccccc}
\hline Condições de Secagem & $\mathrm{T}\left({ }^{\circ} \mathrm{C}\right)$ & $\Delta \mathrm{H}\left(\mathrm{kJ} \cdot \mathrm{mol}^{-1}\right)$ & $\Delta \mathrm{G}\left(\mathrm{kJ} \cdot \mathrm{mol}^{-1}\right)$ & $(\Delta \mathrm{S})\left(\mathrm{kJ} \mathrm{mol}{ }^{-1}\right)$ \\
\hline Bancada & $28,5 \pm 1,01$ & 82,7535 & 129,8949 & $-0,1560$ \\
Sombra & $32,3 \pm 0,387$ & 82,7219 & 130,4879 & $-0,1561$ \\
Sol & $42,8 \pm 4,83$ & 82,6346 & 132,1284 & $-0,1564$ \\
\hline
\end{tabular}


Para entropia $(\Delta S)$, os valores encontrados foram de $-0,1560 ;-0,1561$; e $-0,1564 \mathrm{~kJ} \mathrm{~mol}^{-1}$, para bancada, sombra e sol, respectivamente (Tabela 4). Verifica-se que a entropia diminuiu com o aumento da temperatura. Rodríguez-Bernal et al. (2015) encontraram valores de entropia entre $-0,1500$ e $0,0300 \mathrm{~kJ} \mathrm{~mol}^{-1}$ para a adsorção de água pela polpa de borojó (Alibertia patinoi).

Em estudo, Jideane e Mptokawana (2009) observaram que a entropia tende a reduzir com o aumento da temperatura. Este processo está associado ao fato de que, quando a temperatura diminui, ocorre menor excitação das moléculas de água, assim aumentando o grau de ordem entre o sistema água e fruto.

Ainda na Tabela 4, nota-se que os valores da energia livre de Gibbs $(\Delta G)$ aumentaram (129,8949; 130,4879 e $132,1284 \mathrm{~kJ} \mathrm{~mol}^{-1}$ ), com a elevação da temperatura (bancada, sombra e sol), respectivamente. Dessa forma, conclui-se que o processo de secagem não foi espontâneo, sendo necessária a adição de uma energia proveniente do ar nas fatias do fruto envolvido, para que ocorresse a redução do teor de água.

\section{Conclusão}

O modelo Midilli Modificado é recomendado para representar a cinética de secagem de fatias de banana maçã da cultivar BRS Tropical nas condições de secagem estudadas.

O coeficiente de difusão efetivo aumenta com a elevação da temperatura do ar de secagem, sendo que esta relação pode ser descrita pela equação de Arrhenius.

A energia livre de Gibbs aumenta com o incremento da temperatura, enquanto a entalpia e a entropia decrescem.

\section{Agradecimentos}

Esta pesquisa foi apoiada pelo Instituto Federal Goiano (Campus Ceres), Fundação de Amparo à Pesquisa do Estado de Goiás (FAPEG) e Conselho Nacional de Desenvolvimento Científico e Tecnológico (CNPq).

\section{Referências}

ASABE (2010) Moisture Measurement - Forages: Standard S358.2 DEC1988, R2008. In: American Society of Agricultural And Biological Engineers (Ed.). Standards, Engineering Practices, and Data. St. Joseph: ASABE, 684-685p.

Babalis SJ, Belessiotis VG (2004) Influence of the drying conditions on the drying constants and moisture diffusivity during the thin-layer drying of figs. Journal of Food Engineering 65(3): 449-458.
Bakhtavar MA, AFZAL I, Basra SMA (2019) Moisture adsorption isotherms and quality of seeds stored in conventional packaging materials and hermetic super bag. Plos One 14(2): 1-11.

Brooker DB, Bakker-Arkema FW, Hall CW (1992) Drying and storage of grains and oilseeds. Westport: The AVI Publishing Company, 450p.

Corrêa PC, Botelho FM, Botelho SDCC, Goneli ALD (2014) Isotermas de sorção de água de frutos de Coffea canephora. Revista Brasileira de Engenharia Agrícola e Ambiental [s.I.] 18(10): 1047-1052.

Corrêa PC, Oliveira GHH, Botelho FM, Goneli ALD, Carvalho FM (2010) Modelagem matemática e determinação das propriedades termodinâmicas do café (Coffea arabica L.) durante o processo de secagem. Revista Ceres 57(5): 595-601.

Corrêa PC, Reis MFT, Oliveira GHH De, Oliveira APLR De, Botelho FM (2015) Moisture desorption isotherms of cucumber seeds: modeling and thermodynamic properties. Journal of Seed Science 37(1): 218-225.

Corrêa PC, Resende O, Martinazzo AP, Goneli ALD, Botelho FM (2007) Modelagem matemática para a descrição do processo de secagem do feijão (Phaseolus vulgaris L.) em camadas delgadas. Engenharia Agrícola. 27(1): 501-510.

EMBRAPA (2017) Base de Dados dos Produtos: Banana, Disponível em: <http://www.cnpmf.embrapa.br/Base_de_Dados/index _pdf/dados/brasil/banana/b1_banana.pdf $>$. Acesso em: 12 ago. 2018.

Goneli ALD, Corrêa PC, Afonso JPC, Oliveira GHH (2009) De Cinética de secagem dos grãos de café descascados em camada delgada. Revista Brasileira de Armazenamento 1(64): 73.

Goneli ALD, Vieira MC, Vilhasanti HDCB, Gonçalves AA (2014) Modelagem matemática e difusividade efetiva de folhas de aroeira durante a secagem. Pesquisa Agropecuária Tropical 44(1): 56-64.

Gouveia JPGD, Nascimento JD, Almeida FDAC, Silva MMD, Farias EDS, Silva FLHD (2004) Modelos matemáticos para ajuste das isotermas de dessorção da polpa de banana da variedade prata. Engenharua Agrícola 24(3): 799-806.

Jangam SV, Law CL, Mujumdar (2010) Drying of foods, vegetables and fruits. Singapore: National University of Singapore, 232p.Jideani VA, Mpotokwana SM (2009) Modeling of water absorption of botswana bambara varieties using Peleg's equation. Journal of Food Engineering 92(1): 182-188. 
Jideani V, Mpotokwane SM (2009) Modeling of water absorption of Botswana Bambara varieties using Peleg's equation. Journal of Food Engineering92(2):182 - 188.

Karizaki VM (2016) Kinetic modeling and determination of mass transfer parameters during cooking of rice. Innovative Food Science and Emerging Technologies 38(1): 131-138.

Kim H, Kim J (2017) Kinetics and thermodynamics of microwave-assisted drying of paclitaxel for removal of residual methylene chloride. Process Biochemistry 56(1): 163-170.

Leite ALMP, Silva FS Da, Porto AG, Piasson D, Santos PD (2015) Contração volumétrica e cinética de secagem de fatias de banana variedade Terra. Pesquisa Agropecuária Tropical 45(2): 155-162.

Morais SJS, Devilla IA, Ferreira DA, Teixeira IR (2013) Modelagem matemática das curvas de secagem e coeficiente de difusão de grãos de feijão-caupi (Vigna unguiculata (L.). Revista Ciência Agronômica 44(3): 455-463.

Oliveira GHH, Corrêa PC, Araújo EF, Valente DSM, Botelho FM (2010) Desorption isotherms and thermodynamic properties of sweet corn cultivars (Zea mays L.). International Journal of Food Science \& Technology 45(1): 546-554.

Radünz LL, Amaral ASD, Mossi AJ, Melo EDC, Rocha RP (2011) Avaliação da cinética de secagem de carqueja. Engenharia na agricultura 19(1): 19-27.
Rodríguez BJM, Flores AE, Lizarazo MC, Bonilla E, Pascual PLA, Gutierréz LG, Quintanilla CMX (2015) Moisture adsorption isotherms of the borojó fruit (Borojoa patinoi. Cuatrecasas) and gum arabic powders. Food and Bioproducts Processing 94(1): 187198.

Siqueira VC, Resende O, Chaves TH (2012) Drying kinetics of jatropha seeds. Revista Ceres [s.I.] 59(2): 171-177.

Sousa FC, Martins JJA, Rocha APT, Gomes JP, Pessoa T, Martins JN (2015) Predição de modelos sobre a cinética de secagem de folhas de Ziziphus joazeiro Mart. Revista Brasileira de Plantas Medicinais [s.l.] 17(2): 195-200.

Viganó J, Azuara E, Telis VRN, Beristain Cl, Jiménez M, Telis RJ (2012) Role of enthalpy and entropy in moisture sorption behavior of pineapple pulp powder produced by different drying methods. Thermochimica Acta 528(1): 63-71.

Zeymer JS, Corrêa PC, Oliveira GHH De, Baptestini FM, Freitas RCP (2017) Desorption isotherms of Lactuca sativa seeds. Revista Brasileira de Engenharia Agrícola e Ambiental 21(1): 568-572. 